\section{Morbidade materna extremamente grave a partir dos registros de internação hospitalar do Sistema Único de Saúde: algoritmo para identificação dos casos}

\section{Extremely severe maternal morbidity in Brazilian National Health System hospital registers: an algorithm for identification of cases}

Maria da Consolação Magalhães 1

Carlos Eduardo Raymundo 2

Maria Teresa Bustamante-Teixeira 3

\begin{abstract}
Objectives: to identify codes for constant procedures in the Brazilian National Health System's Hospital Information System (SIH-SUS) deemed to be cases of Extremely Severe Maternal Morbidity (MMEG) and to develop an algorithm to manage/prepare an AIH database, with a view to identifying cases of MMEG in this database.

Methods: the data used were from the SIH-SUS supplied by the Juiz de Fora Health Secretary and refer to admissions to SUS hospitals between 2006 and 2007. The study covered all admissions where the principal diagnosis involved all of Chapter $X V$ of CID10 - complications of pregnancy, miscarriage, delivery and puerperium and/or admissions to hospital involving obstetric procedures. For identification of SIH-SUS procedures deemed to be MMEG, the criteria proposed by the WHO were used. The algorithm was developed using Microsoft Access.

Results: 326 cases of MMEG were found among the 8620 women selected, constituting a rate of 37.8/1000 women. The most frequent procedures were transfusion of blood products, "a longer stay" and severe pre-eclampsia/eclampsia, with a prevalence of 15.7/1000, 9.5/1000 and 8.2/1000, respectively.

Conclusions: the algorithm used may optimize the use of the SIH-SUS for identifying cases of MMEG and generating information for maternal morbidity and mortality surveillance services and the evaluation of obstetric care.
\end{abstract}

Key words Medical informatics, Health evaluation, Morbidity, Hospital Information Systems, Pregnancy complications

\author{
1 Secretaria de Saúde de Juiz de Fora. Rua Halfeld, 1400. $3^{\circ}$ andar. \\ Centro. Juiz de Fora, MG, Brasil. CEP: 36.013-000. \\ E-mail: consolamagalhaes@gmail.com \\ 2 Instituto de Medicina Social. Universidade do Estado do Rio de \\ Janeiro. Rio de Janeiro, RJ, Brasil. \\ 3 Universidade Federal de Juiz de Fora. Juiz de Fora, MG, Brasil.
}

Resumo

Objetivos: identificar os códigos de procedimentos constantes no Sistema de Informação Hospitalar - Sistema Único de Saúde (SIH-SUS) considerados como Morbidade Materna Extremamente Grave (MMEG) e construir um algoritmo para o manejo/preparação da base de dados de Autorização de Internação Hospitalar visando a captura dos casos de MMEG neste banco de dados.

Métodos: utilizaram-se os dados do SIH-SUS fornecidos pela Secretaria de Saúde de Juiz de Fora e referem se as internações ocorridas no SUS no período de 2006-2007. Foram selecionadas todas as internações cujo diagnóstico principal compreendia todo o capítulo XV da Classificação Internacional de Doenças (CID10) - Complicações da gravidez, aborto, parto e puerpério elou internações em que procedimentos obstétricos foram realizados. Para identificação dos procedimentos no SIH-SUS, considerados como MMEG, adotaram-se os critérios propostos pela Organização Mundial da Saúde. O algoritmo foi desenvolvido no software Microsoft Access.

Resultados: foram capturados 326 casos de MMEG entre as 8620 mulheres selecionadas, perfazendo uma taxa de 37,8/1000 mulheres. Os procedimentos mais frequentes foram transfusão de hemoderivados, "permanência a maior" e préeclampsia graveleclâmpsia, com prevalências de 15,7/1000, 9,5/1000 e 8,2/1000, respectivamente.

Conclusões: o algoritmo utilizado pode otimizar o uso do SIH-SUS para a captação dos casos de MMEG e gerar informações para os serviços de vigilância da morbimortalidade materna e avaliação de cuidados obstétricos.

Palavras-chave Informática em saúde, Avaliação em saúde, Morbidade, Sistemas de Informação Hospitalar, Complicações na gravidez 


\section{Introdução}

A Organização Mundial da Saúde (OMS) define que um caso de near miss materno ou Morbidade Materna Extremamente Grave (MMEG) é aquele em que a mulher quase foi a óbito por complicações que ocorreram durante a gestação, parto ou até 42 dias após o término da gestação, mas sobreviveu. ${ }^{1}$ Os critérios de MMEG tem variado entre os autores, ${ }^{2-7}$ mas, recentemente, a OMS ${ }^{1}$ definiu alguns destes para identificação de casos de MMEG: critérios clínicos (choque, distúrbios da coagulação, acidente cerebrovascular, perda de consciência por mais de 12 horas, entre outros); critérios laboratoriais (trombocitopenia aguda, creatinina $>3,5 \mathrm{md} / \mathrm{dL}$ e outros) e procedimentos (diálise, histerectomia puerperal, uso contínuo de drogas vasoativas, entre outros).

No Brasil, os sistemas de informação em saúde disponíveis contam com grande número de dados que poderiam contribuir para o estudo da morbimortalidade materna. O Sistema de Informação Hospitalar - Sistema Único de Saúde (SIH-SUS), que se refere aos internamentos registrados pelo SUS, seria um deles. Vários estudos8-11 de concordância de registros de procedimentos e de diagnósticos têm demonstrado a confiabilidade das informações nele contidas. Esses registros têm sido utilizados ainda para captura das internações devido às condições sensíveis aos cuidados na atenção primária à saúde 22,13 e também como fonte de identificação de doenças de notificação compulsória desde 2005.14

Este trabalho objetivou identificar os códigos de procedimentos constantes no SIH-SUS considerados como MMEG e construir um algoritmo para manejo/preparação da base de dados e captura dos casos neste banco de dados.

\section{Métodos}

Os dados do Sistema de Informação Hospitalar (SIH-SUS) foram fornecidos pela Secretaria de Saúde de Juiz de Fora e referem se as internações ocorridas no SUS no período de 2006 e 2007. Inicialmente foram selecionadas, na Tabela TB-AIH, todas as internações cujo diagnóstico principal compreendia o Capítulo XV da CID-10 (Complicações da gravidez, aborto, parto e puerpério) e/ou internações, fora deste capítulo, em que constavam a realização de procedimentos obstétricos, identificados pelos códigos de procedimentos iniciados por 34, 35 e 69 no SIH-SUS. 15

O SIH-SUS fornece diversas tabelas que armazenam, cada uma delas, dados relativos a carac- terísticas do usuário, procedimentos e atos realizados na internação, entre outros. A Tabela TB-AIH fornece dados gerais relativos à internação como o número da Autorização de Internação Hospitalar (AIH), identificação do usuário e do hospital, datas de internação e saída, número de dias de UTI, diagnóstico principal e secundário, procedimento solicitado e realizado, médico responsável, e outros. Essa contém todas as internações do período. A Tabela TB-HSP abrange os atos profissionais realizados em cada internação, identificação do profissional, os respectivos valores e quantidades de atos, entre outros. A Tabela TB-HPE apresenta os procedimentos especiais realizados. São utilizados os mesmos códigos para atos profissionais, procedimentos realizados e procedimentos especiais. Nas Tabelas TB-HSP e TB-HPE o número da AIH se repete cada vez que houver um procedimento para a internação registrada na TB-AIH. Se o paciente foi submetido a dez procedimentos ou atos profissionais, por exemplo, a AIH estará repetida este número de vezes nas tabelas referidas.

Para identificação dos procedimentos considerados como MMEG, foram selecionadas as variáveis HAH-PROC-R (procedimento realizado), HSP-ATO (ato profissional) e HPE-PROC (procedimentos especiais) nas Tabelas TB-AIH, TB-HSP e TB-HPE, respectivamente, considerando os procedimentos identificados como MMEG a partir dos critérios propostos por Say et al. ${ }^{1}$ apresentados na Tabela 1.

$\mathrm{O}$ número da $\mathrm{AIH}$, comum em todas as tabelas, foi utilizado para junção e composição da base de dados para análise (BDA). O algoritmo para junção das tabelas disponíveis no banco do SIH-SUS pode ser visto na Figura 1. A construção da BDA se inicia com a união das tabelas TB-AIH, TB-HPE e TBHSP, referentes aos anos de 2006 e 2007, respectivamente. O próximo passo caracteriza-se pela junção destas três tabelas em um único arquivo, contendo ainda as variáveis relativas aos critérios de morbidade conforme descrito na Figura 1. A seguir são realizadas duas rotinas de exclusão de duplicidades (total 68.213 registros, 7377 duplicidades). A primeira faz a eliminação de registros a partir dos campos-chave: hsp_num_ai (número da AIH), hsp_ato (código do ato profissional), hah_nome_p (nome do usuário), hah_dt_nas (data de nascimento). Já a segunda rotina exclui registros que atendem aos campos-chave hsp_num_ai, hah_nome_p e hah_dt_nas, criando a tabela temporária "tabtemp2" (total 60.836, 52.207 duplicidades). A seguir é feito uma inspeção manual para busca de duplicidades que não foram detectadas pela rotina, a qual identificou mais nove duplicidades $(0,1 \%)$, totalizando 
Procedimentos ou condições clínicas, com os respectivos códigos, considerados indicativos de morbidade materna extremamente grave no banco de dados do Sistema de Informação Hospitalar - Sistema Único de Saúde (SIH-SUS).

\section{Procedimentos ou condições clínicas}

Admissão em UTI

Albumina Humana

Cardioversão

Choque anafilático

Choque cardiogênico no adulto

Choque hipovolêmico no adulto

Choque séptico

Complicações de procedimentos cirúrgicos ou médicos

Concentrado de hemácias

Concentrado de leucócitos

Concentrado de plaquetas

Defeitos da coagulação

Eclampsia

Edema agudo de pulmão

Hemorragias na gravidez

Histerectomia puerperal

Implante de cardiodesfibrilador

Implante de marcapasso

Infecção parede abdominal

Infecção parto e puerperio

Instalação de assistência circulatória

Insuficiência renal aguda

Insuficiência respiratória aguda

Intercorrência clinica de atendimento secundário em gestante de alto risco

Intercorrência clínica na gravidez em gestante de alto risco

Intercorrencia obstétrica atendimento secundário a gestante de alto risco

Intercorrencia obstétrica na gravidez em gestante de alto risco

Laparotomia exploradora

Laparotomia para histerorrafia

Mastite

Outras histerectomias

Peritonite pós cesareana

Permanência a maior

Pielonefrite

Plasma individual

Preeclampsia grave

Púrpura trombocitopenica

Ressutura de parede abdominal

Sangue total

Septicemia

Tireoidite

Tireotoxicose

Tratamento conservador da hemorragia cerebra
Códigos correspondentes no SIH-SUS

$96001011,96001020,96001038$

$96002018,96002026,96002034$

$92037011-92037992$

004001028

86300016,86300015

77500199

77500245

77500482

91500141

94006016

99004013

94002010

79300057

69000069

77300149

69000140

35011017

48010421

48010405

69000034

69000093

48010170

80300057,8050056

76300188,76500233

69500029

69500010

35032014

35031018

33016119

35012013

69000026

$34009035,34010033,34011030$

$34014039,34017038,3474124,34709037$.

34710035,34717030

69000042

99003015 a 99003996

80300073,80500072

94005010

69000050

79300065

33022119

94007012

74500244

8250037

82300020

40202003

* baseado nos critérios propostos pela OMS. ${ }^{1}$ 
8620 mulheres. Na última etapa são criadas as variáveis número e tempo de internação total por usuária armazenadas na tabela temporária "tabtemp3". Estas duas últimas tabelas são agregadas formando o banco de análise final (BDA). A sintaxe do Microsoft Access para identificação dos casos de MMEG no banco de dados do SIH-SUS está disponível em http://www.ufjf.br/nates/files/ 2008/09/Rotina-para-identifica\%C3\%A7\%C3\%A3o -dos-casos-de-Morbidade-Materna-Extremamente-G rave-MMEG.pdf

O presente projeto foi submetido ao comitê de ética de pesquisa da Universidade Federal de Juiz de Fora e aprovado pelo parecer $n^{\circ} 468 / 2007$.

\section{Resultados}

A Tabela 2 apresenta os procedimentos/condições selecionados. Entre as 8620 foram identificadas 326 mulheres com morbidade materna extremamente grave, ou seja, uma taxa de MMEG de 37,8/1000 mulheres. Os procedimentos mais frequentes foram: transfusão de hemoderivados, "permanência a maior" (procedimento especial autorizado e registrado no sistema quando o período de internação ultrapassa o dobro da permanência prevista na tabela de procedimentos) e pré-eclampsia grave/eclâmpsia, com prevalências de morbidade específicas de 15,7/1000, 9,5/1000 e 8,2/1000, respectivamente.

\section{Figura 1}

Algoritmo para adequação da base de dados de identificação de casos de Morbidade Materna Extremamente Grave (MMEG).

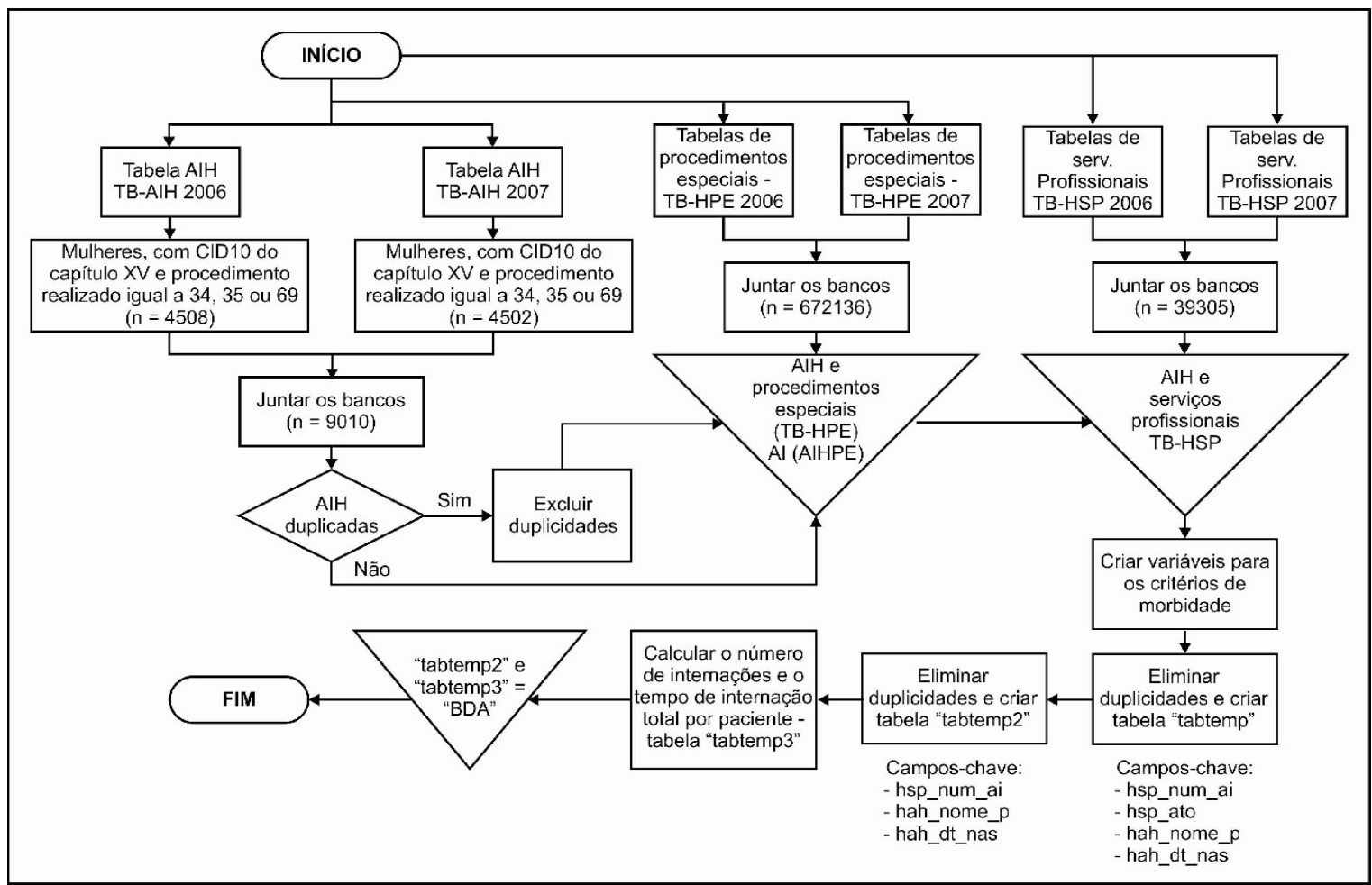


Procedimentos ou diagnósticos considerados como critérios de morbidade materna extremamente grave (MMEG) em mulheres internadas pelo Sistema Único de Saúde/Juiz de Fora, 2006-2007.

\begin{tabular}{|c|c|c|c|}
\hline Variáveis & N & $\%$ & TX. MMEG \\
\hline \multicolumn{4}{|l|}{ Diagnóstico e/ou condições clínicas } \\
\hline Complicações de procedimentos cirúrgicos ou médicos & 11 & 3,4 & 1,3 \\
\hline Eclampsia/ Preeclampsia grave & 71 & 21,8 & 8,2 \\
\hline Hemorragias na gravidez & 10 & 3,1 & 1,2 \\
\hline $\begin{array}{l}\text { Intercorrência clinica /obstétrica de atendimento secundário } \\
\text { em gestante de alto risco }\end{array}$ & 28 & 8,6 & 3,4 \\
\hline \multicolumn{4}{|l|}{ Procedimentos } \\
\hline Admissão em UTI & 2 & 0,6 & 0,2 \\
\hline Histerectomia & 10 & 3,0 & 1,2 \\
\hline Transfusão de hemoderivados* & 136 & 41,7 & 15,7 \\
\hline Total de mulheres com MMEG & 326 & ** & 37,8 \\
\hline
\end{tabular}

*plasma, sangue total, hemácias, albumina humana; ** os percentuais não somam $100 \%$ porque uma mulher pode ter apresentado mais de um diagnóstico ou procedimento.

\section{Discussão}

A MMEG vem ganhando importância devido à queda na razão de mortalidade materna e buscar formas de identificar estas ocorrências torna-se fundamental para o monitoramento da saúde materna. Algumas pesquisas têm sido realizadas a partir de registros em prontuários hospitalares para identificação dos casos de MMEG.2-7 Embora sejam ideais, na rotina dos serviços de saúde, especialmente para vigilância da morbimortalidade esta busca se torna pouco produtiva. Neste sentido a utilização dos dados secundários constitui-se em fonte importante de informação que poderia ser incorporada na rotina da vigilância da saúde materna. $\mathrm{O}$ algoritmo proposto demonstrou ser eficiente para a construção do banco de dados de procedimentos e na identificação dos casos de

\section{Referências}

1. Say L, Souza JP, Pattinson RC. WHO working group on maternal mortality and morbidity classifications. Maternal near miss - towards a standard tool for monitoring quality of maternal health care. Best Pract Res Clin Obstet Gynaecol. 2009; 23: 287-96.

2. Sheikh L, Zuberi NF, Riaz R, Rizvi JH. Massive primary postpartum haemorrhage: setting up standards of care. J Pak Med Assoc. 2006; 56: 26-31.

3. Weeks A, Lavender T, Nazziwa E, Mirembe F. Personal accounts of 'near-miss' maternal mortalities in Kampala, Uganda. BJOG. 2005; 112: 1302-7.

4. Murphy DJ, Charlett P. Cohort study of near-miss maternal morbidade materna, embora tenha sido pouco sensível para captação de óbitos maternos, uma vez que grande parte destes pode ocorrer no puerpério ou em serviços de urgência/emergência. Nos casos de reinternação, nem sempre o motivo da internação faz referência ao período gravídico puerperal. No entanto, a identificação dos casos de MMEG podem constituir-se em fonte importante para os comitês de investigação de morte materna, agilizando seu trabalho.

O algoritmo aqui proposto visa otimizar o uso do SIH-SUS para a captação dos casos de MMEG e, se adotado pelos gestores, poderia gerar automaticamente e em tempo hábil informações para os serviços de vigilância da morbimortalidade materna, permitindo a avaliação e monitoramento de cuidados obstétricos. mortality and subsequent reproductive outcome. Eur J Obstet Gynecol Reprod Biol. 2002; 10: 102: 173-8.

5. Waterstone M, Bewley S, Wolfe C. Incidence and predictors of severe obstetric morbidity: case-control study. BMJ. 2001; 322 (7294): 1089-94.

6. Mantel GD, Buchmann E, Rees H, Pattinson RC. Severe acute maternal morbidity: a pilot study of a definition for a near-miss. Br J Obstet Gynaecol. 1998; 105: 985-90.

7. Geller SE, Rosenberg D, Cox SM, Brown ML, Simonson L, Driscoll CA, Kilpatrick SJ. The continuum of maternal morbidity and mortality: factors associated with severity. Am J Obstet Gynecol. 2004; 191: 939-44. 
8. Mathias TAF, Soboll MLMS. Confiabilidade de diagnósticos nos formulários de autorização de internação hospitalar. Rev Saúde Pública. 1998; 32: 526-32.

9. Lebrao ML. Análise da fidedignidade dos dados estatísticos hospitalares disponíveis na Secretaria de Estado da Saúde de São Paulo em 1974. Rev Saúde Pública. 1978; 12: 234 49 .

10. Veras CMT, Martins MS. A confiabilidade dos dados nos formulários de autorizaçäo de internaçäo hospitalar (AIH), Rio de Janeiro, Brasil. Cad Saúde Pública. 1994; 10: 33955.

11. Bittencourt AS, Camacho LAB, Leal MC. A qualidade da informação sobre o parto no Sistema de Informações Hospitalares no Município do Rio de Janeiro, Brasil, 1999 a 2001. Cad Saúde Pública. 2008; 24: 1344-54

12. Alfradique ME, Bonolo PF, Dourado I, Lima-Costa MF, Macinko J, Mendonça CS, Oliveira VB, Sampaio FLR, De Simoni $C$, Turci MA. Internações por condições sensíveis à atenção primária: a construção da lista brasileira como ferramenta para medir o desempenho do sistema de saúde (Projeto ICSAP - Brasil). Cad Saúde Pública. 2009; 25: 1337-49.
13. Moreno AB, Caetano R, Coeli CM, Ribeiro LC, Bustamante-Teixeira MT, Camargo Jr KR, Campos EMS. Internações hospitalares por condições sensíveis à atenção ambulatorial: algoritmo de captura em registro integrado de saúde. Cad Saúde Colet. 2009; 17: 409-16.

14. Brasil. Ministério da Saúde. Secretaria de Atenção à Saúde. Secretaria de Vigilância em Saúde. Portaria MS, SAS, SVS $\mathrm{n}^{\circ} 20$ de 25 de maio de 2005. Estabelece que todas as Autorizações de Internação Hospitalar - AIH com agravos de notificação compulsória (ANC) identificadas através da CID10 fiquem bloqueadas até a avaliação pelo Serviço de Vigilância Epidemiológica. Diário Oficial da União, Brasília, DF, 30 maio 2005. Sec. 1, p. 77.

15. Brasil. Ministério da Saúde. Secretaria de Atenção à Saúde. Departamento de Regulação. Avaliação e Controle. Manual do Sistema de Informação Hospitalar/Atualização. Brasília, DF; 2006.

Recebido em 31 de maio de 2012

Versão final apresentada em 14 de dezembro de 2012

Aprovado em 17 de janeiro de 2013 